\title{
Fgf8-deficient mice compensate for reduced GnRH neuronal population and exhibit normal testicular function
}

\author{
Wei Zhang, Joshua I. Johnson and Pei-San Tsai* \\ Department of Integrative Physiology, Center for Neuroscience, University of Colorado Boulder, Boulder, CO, USA
}

OPEN ACCESS

Edited by:

Yong Zhu,

East Carolina University, USA

Reviewed by:

T. John Wu,

Uniformed Services University of the Health Sciences, USA

Paolo Giacobini,

Université Lille 2, France

*Correspondence:

Pei-San Tsai,

Department of Integrative Physiology,

University of Colorado Boulder,

114 Clare Small, Boulder,

CO 80309-0354, USA

pei-san.tsai@colorado.edu

Specialty section: This article was submitted to

Experimental Endocrinology, a section of the journal

Frontiers in Endocrinology

Received: 04 June 2015 Accepted: 07 September 2015 Published: 22 September 2015

Citation:

Zhang W, Johnson Jl and Tsai P-S (2015) Fgf8-deficient mice compensate for reduced GnRH neuronal population and exhibit normal testicular function.

Front. Endocrinol. 6:151. doi: 10.3389/fendo.2015.00151
Gonadotropin-releasing hormone $(\mathrm{GnRH})$ is critical for the onset and maintenance of reproduction in vertebrates. The development of $\mathrm{GnRH}$ neurons is highly dependent on fibroblast growth factor (Fgf) signaling. Mice with a hypomorphic Fgf8 allele (Fgf8 Het) exhibited a 50\% reduction in $\mathrm{GnRH}$ neuron number at birth. Female Fgf8 Het mice were fertile but showed significantly delayed puberty. However, it was unclear if these mice suffered additional loss of GnRH neurons after birth, and if male Fgf8 Het mice had normal pubertal transition and testicular function. In this study, we examined postnatal $\mathrm{GnRH}$ neuron number and hypothalamic GnRH content in Fgf8 Het mice from birth to 120 days of age. Further, we examined seminal vesicle and testicular growth, testicular histology, and circulating luteinizing hormone (LH) around and after pubertal transition. Our results showed that $\mathrm{GnRH}$ neuron numbers were significantly and consistently reduced in Fgf8 Het mice of both sexes in all ages examined, suggesting these animals were born with an inherently defective GnRH system, and no further postnatal loss of $\mathrm{GnRH}$ neurons had occurred. Despite an innately compromised $\mathrm{GnRH}$ system, male and female Fgfo mice exhibited normal levels of immunoassayable hypothalamic $\mathrm{GnRH}$ peptide at all ages examined except on 60 days of age, suggesting increased $\mathrm{GnRH}$ synthesis or reduced turnover as a compensatory mechanism. Fgf8 Het males also had normal seminal vesicle and testicular mass/body mass ratios, testicular histology, and circulating $\mathrm{LH}$. Overall, our data speak to the extraordinary ability of a $\mathrm{GnRH}$ system permanently compromised by developmental defect to overcome pre-existing deficiencies to ensure pubertal progression and reproduction.

Keywords: GnRH, Fgf8, hypogonadotropic hypogonadism, development, puberty, testes

\section{Introduction}

Neurons that synthesize and secrete gonadotropin-releasing hormone $(\mathrm{GnRH})$ are critical to vertebrate reproduction. The functional network of GnRH neurons develops via a multi-step process that includes GnRH neuronal fate specification in the nose, migration to the forebrain, and axon targeting to the median eminence. GnRH released from the mature GnRH system is ultimately responsible for the stimulation of pituitary gonadotropin secretion, and hence gonadal maturation and reproduction. The development of $\mathrm{GnRH}$ neurons is orchestrated by a multitude of signaling factors (1-3). The importance of these factors is underscored by observations that loss-of-function mutations in many of these signaling molecules could lead to 
hypogonadotropic hypogonadism $(\mathrm{HH})$ in humans, a disorder characterized by low circulating gonadotropins and absent puberty.

Fibroblast growth factor (Fgf) signaling is a large family of genes that comprises 22 ligands, 4 receptors, several ligand and receptor splice variants, and genes synexpressed with Fgf ligands (4-13). Fgf signaling is indispensable for the development, survival, and maturation of the central nervous system $(9,13)$. Mounting evidence from human studies reveals loss-of-function mutations on Fgfreceptor ( Fgfr) 1, Fgf8, Fgf17, and synexpression genes such as IL17RD, DUSP6, SPRY4, and FLRT3 are causal to $\mathrm{HH}$ (14-16). Studies in mice confirm that many of the Fgf signaling genes are expressed prominently in the olfactory placode, the birthplace of GnRH neurons $(16,17)$, and their deficiencies could lead to severely reduced GnRH neuronal population (15, 18-22). Of these, Fgf8 is particularly critical for the development of GnRH neurons. Heterozygous Fgf8 hypomorphic mice ( $F g f 8$ Het) exhibit a 50\% reduction in GnRH neurons at birth (20), whereas newborn homozygous $\mathrm{Fgf8}$ hypomorphic mice exhibit a complete elimination of GnRH neurons due to fate specification failure (20).

Although the role of Fgf8 signaling in GnRH neuronal development has been reported, two questions remain unanswered. First, deficiency in Fgfr3, one of the cognate receptors for Fgf8 (23), results in the postnatal loss of GnRH neurons without affecting their development $(20,21)$. It is currently unclear if Fgf8 also contributes to the postnatal maintenance of the $\mathrm{GnRH}$ system in addition to its early effects on the genesis of $\mathrm{GnRH}$ neurons. Second, an interesting paradox is that while a fraction of humans with $F g f 8$ haploinsufficiency experiences absent puberty and infertility $(15,24), F g f 8$ Het mice consistently go through puberty and propagate offspring (25). This raises the possibility that the GnRH system of Fgf8 Het mice may compensate for the loss of GnRH neurons by upregulating the production of GnRH. Interestingly, although Fgf8 Het female mice are fertile, they exhibit delayed vaginal opening (VO) and first estrus (26). It is at present unclear if $\mathrm{Fgf8} \mathrm{Het}$ male mice exhibit normal gonadal function during and after puberty.

The objectives of this study are twofold. First, we examine if Fgf8 Het mice suffer an additional loss of GnRH neurons after birth, and if a smaller GnRH neuronal population resulting from Fgf8 deficiency could produce normal levels of the GnRH peptide to offset the overall neuronal loss. Second, by measuring the growth and maturation of testes and seminal vesicle (SV) as well as circulating luteinizing hormone (LH) level, we examine if male Fgf8 Het mice undergo normal pubertal progression compared to wildtype (WT) controls. Our results show that $\mathrm{Fgf8}$ deficiency obliterates half of GnRH neuronal population prenatally and permanently in both sexes, but all mice compensate for this innate GnRH system deficit by increasing the levels of the GnRH peptide before, during, and after puberty with only some rare exceptions. Further, male Fgf8 Het mice have normal circulating LH and undergo testicular growth and spermatogenesis comparable to WT controls. Our results highlight the ability of an inherently defective murine $\mathrm{GnRH}$ system to overcome a reduction in its neuronal population in order to facilitate pubertal transition and support reproduction in adulthood.

\section{Materials and Methods}

\section{Animals}

Fgf8 Het mice (129P2/OlaHsd ${ }^{\star}$ CD-1) (25) were initially obtained from the Mouse Regional Resource Centers (Davis, CA, USA) and maintained in the University of Colorado animal facility under a 12L:12D cycle and fed rodent chow and water ad libitum. $\mathrm{Fgf8}$ hypomorphic mice were generated by the insertion of a neo cassette in the non-coding region of the $F g f 8$ gene, resulting in the abnormal splicing of $\mathrm{Fgf8}$ transcript. Homozygous $\mathrm{Fg} f 8$ hypomorphs showed a 54\% decrease in functional Fgf8 message, died within $24 \mathrm{~h}$ of birth (25), and were not used in this study. Fgf8 Het mice, however, were viable and propagated offspring. WT and Fgf 8 Het used in this study were derived from the breeding of Fgf8 Het $\times F g f 8$ Het or WT $\times F g f 8$ Het. The day of birth was designated as postnatal day (PN) 0 . Mice were weaned at approximately PN20 and housed separately by sex after weaning. All animals were genotyped by polymerase chain reaction of genomic DNA isolated from tail biopsies. All animal procedures complied with protocols approved by the Institutional Animal Care and Use Committee at the University of Colorado.

\section{GnRH Immunohistochemistry and Quantification of GnRH Neurons}

GnRH immunohistochemistry (IHC) was carried out as described previously $(20,21)$. Briefly, the brain region containing the preoptic area (POA) was dissected by blocking anteriorly at the caudal border of the olfactory bulbs and posteriorly at the optic chiasm. Blocked tissues were immersion-fixed in $5 \%$ acrolein in $0.1 \mathrm{M}$ phosphate buffer for $6 \mathrm{~h}$ (for PN0) or overnight (for older animals), and then cryoprotected in $30 \%$ sucrose. Fixed brain tissues were sectioned at $50-\mu \mathrm{m}$ thickness in a cryostat and transferred into a cryoprotectant containing $30 \%$ sucrose, $1 \%$ polyvinylpyrolidone, and $30 \%$ ethylene glycol in $0.05 \mathrm{M}$ phosphate buffer, washed in $0.1 \mathrm{M}$ phosphate-buffered saline (PBS), and incubated with $1 \%$ sodium borohydride in PBS for $40 \mathrm{~min}$ to quench the activity of residual acrolein. Sections were then washed extensively in PBS, incubated with $1 \% \mathrm{H}_{2} \mathrm{O}_{2}$ in $\mathrm{PBS}$ for $15 \mathrm{~min}$ to quench the endogenous peroxidase activity, and then washed in PBS containing $0.4 \%$ Triton X (PBST). All sections were incubated with a polyclonal antibody against GnRH (LR5; from Dr. Robert Benoit, McGill University Health Center, Montréal, QC, Canada; 1:20,000) diluted in PBST containing $4 \%$ normal donkey serum and $10 \%$ normal horse serum for 5 days at $4^{\circ} \mathrm{C}$. After 5 days, sections were washed in PBST, incubated with a biotinylated donkey anti-rabbit IgG (1:500) for $1.5 \mathrm{~h}$, washed in PBST, incubated with an avidin-biotinperoxidase complex (Vector Labs, Burlingame, CA, USA) for $1 \mathrm{~h}$, washed in PBST, and visualized using diaminobenzidine as a chromagen. Sections were then mounted onto gelatin-coated slides, dehydrated through graded ethanol series and coverslipped. GnRH neurons within set distances anterior $(1500 \mu \mathrm{m}$ for PN0-10, $2100 \mu \mathrm{m}$ for PN20-35, $2450 \mu \mathrm{m}$ for PN60, $2700 \mu \mathrm{m}$ for PN120) and posterior (500 $\mu \mathrm{m}$ for PN0, $650 \mu \mathrm{m}$ for PN10, $700 \mu \mathrm{m}$ for PN20-35, $900 \mu \mathrm{m}$ for PN60, $1100 \mu \mathrm{m}$ for PN120) to the organum vasculosum of the lamina terminalis (OVLT) were scored for GnRH neurons by an investigator blind to the 
identity of the slides. Although these set distances may exclude a few of the most anterior and posterior GnRH neurons, this method ensured that the number of sections quantified was consistent among all animals within the same age group. Only positively stained cells that appeared neuronal and exhibited clear nuclei were scored.

\section{GnRH Radioimmunoassay}

Hypothalami were removed by blocking anteriorly at the optic chiasm, posteriorly at the anterior border of the mammillary body, sagittally by two cuts along the lateral borders of the hypothalamus, and dorsally with one cut to remove the cortex. Tissues were immediately flash-frozen on pulverized dry ice and stored at $-70^{\circ} \mathrm{C}$ until further processing. For $\mathrm{GnRH}$ extraction, tissues were sonicated in $0.1 \mathrm{M}$ phosphate buffer on ice, boiled for $2 \mathrm{~min}$, flash-frozen on dry ice and stored at $-70^{\circ} \mathrm{C}$ until $\mathrm{GnRH}$ radioimmunoassay (RIA). Gonadotropin-releasing hormone RIA was carried out using a GnRH-specific antiserum (R1245, provided by T. M. Nett at Colorado State University). Detailed protocol for GnRH RIA was described elsewhere (27). The detection limit was $1.87 \mathrm{pg} /$ tube. The intra- and inter-assay coefficients of variation were 7.3 and $5.0 \%$, respectively.

\section{LH Radioimmunoassay}

Trunk blood from decapitated PN35 and PN60 male WT and Fgf8 Het mice was collected into heparinized tubes and the plasma isolated by centrifugation. Plasma LH was measured using a rat LH RIA as previously described (28). The detection limit was $0.06 \mathrm{ng} / \mathrm{ml}$. The intra- and inter-assay coefficients of variation were 9.3 and $10.1 \%$, respectively.

\section{Seminal Vesicle and Testicular Parameters}

PN20, 35, 40, and $60 \mathrm{WT}$ and Fgf8 Het male mice were weighed before sacrifice. Testes and SV from all animals were dissected and weighed, and all testes were immersion-fixed in Bouin's fixative overnight. Fixed testes were stored in $70 \%$ ethanol, dehydrated in increasing concentrations of ethanol, cleared in Histoclear (National Diagnostics, Atlanta GA, USA), and embedded in paraffin. Serial sections were cut at $12-\mu \mathrm{m}$ coronally on a rotary microtome, mounted on slides and stained with hematoxylin and eosin before dehydration and coverslipping.

\section{Morphometric Analysis}

Eight testicular sections, each five sections from the next, were scored for each animal. For each section, five seminiferous tubules (STs) were randomly selected from each of the following five zones: upper, lower, left, right, and middle portions of the testicular cross-section. Therefore, a total of 200 STs were measured for each testis. Total and luminal diameters of each ST were measured by a calibrated ocular micrometer. Since most STs were elliptical in shape, each total or luminal diameter measurement consisted of an average of two scores: one taken at the widest and one at the narrowest part. The number of ST with open lumen and mature sperm in each scored section was also recorded. Only clear opening with rounded boundary were considered as an open lumen. Mature sperm was identified by the appearance of both the head and the tail in the lumen.

\section{Statistical Analysis}

Differences in GnRH neuron number, hypothalamic GnRH, plasma LH, testicular/body mass and SV/body mass ratios, and histological measurements between genotypes and among ages were analyzed by two-way ANOVA followed by Bonferroni's post hoc test. Differences were considered significant when $P<0.05$. To analyze the percentage of $\mathrm{GnRH}$ neurons lost after birth, GnRH neurons number for each Fgf8 Het mouse was first normalized to the mean of $\mathrm{GnRH}$ neuron numbers in WT mice of same sex and age. The normalized numbers (\% control GnRH neurons) were analyzed by Kruskal-Wallis test followed by Dunn's post hoc test.

\section{Results}

To examine if postnatal loss of GnRH neurons occurred in Fgf8 Het mice, GnRH neurons in PN0, 10, 20, 25, 30, 35, 60, and 120 male and female mice were analyzed (Figures 1 and 2). For males, two-way ANOVA revealed a significant effect of genotype $[F(1,67)=152.5 ; P<0.0001]$, age $[F(6,67)=4.7 ; P=0.0005]$, but no genotype $\times$ age interaction $[F(6,67)=2.2 ; P=0.053]$ on GnRH neuron number in males (Figure 1A). Post hoc test revealed a significant reduction of GnRH neurons in Fgf8 Het males compared to WT males in every age group examined starting at PN0 (Figure 1A). When GnRH neuronal count in Fgf8 Het males was analyzed as percent of $\mathrm{GnRH}$ neurons found in agematched WT males (Figure 2A), Kruskal-Wallis test detected no significant differences across the ages examined $(P=0.14)$, suggesting Fgf8 deficiency did not contribute to postnatal GnRH neurons loss within the first 120 days of birth. For females, two-way ANOVA revealed a significant effect of genotype $[F(1,68)=119.4 ; P<0.0001]$, age $[F(6,68)=3.16 ; P<0.0086]$, but no genotype $\times$ age interaction $[F(6,68)=0.67 ; P=0.67]$ on GnRH neuron number (Figure 1B). Similar to males, post hoc test also revealed a significant reduction of $\mathrm{GnRH}$ neurons in Fgf8 Het females compared to WT females in every age group examined (Figure 1B). When GnRH neuronal count in Fgf8 Het females was analyzed as percent of GnRH neurons found in agematched WT females (Figure 2B), Kruskal-Wallis test, again, detected no significant differences across the ages examined $(P=0.47)$. Averaging all time points examined, male and female Fgf8 Het mice had $55.3 \pm 2.8$ and $59.6 \pm 2.5 \%$, respectively, of the normal complement of GnRH neurons found in WT controls. Representative images of GnRH neurons near the OVLT in PN35 WT and Fgf8 Het mice are shown (Figures 1C-F).

Next, we examined the levels of immunoassayable GnRH peptide in the hypothalami of WT and Fgf8 Het mice at different ages and in both sexes. For males, two-way ANOVA revealed a significant effect of genotype $[F(1,86)=14.93 ; P<0.0002]$, age $[F(6,86)=24.1 ; P<0.0001]$, and genotype $\times$ age interaction $[F(6,86)=2.91 ; P=0.01]$ on hypothalamic GnRH (Figure 3A). Post hoc test revealed a significant reduction of hypothalamic $\mathrm{GnRH}$ in Fgf8 Het males only on PN60 (Figure 3A). For females, two-way ANOVA revealed a significant effect of genotype $[F(1,91)=18.45 ; P<0.0001]$, age $[F(6,91)=15.96 ; P<0.0001]$, and genotype $\times$ age interaction $[F(6,91)=2.48 ; P=0.029]$ on hypothalamic GnRH (Figure 3B). Similar to males, post hoc test 

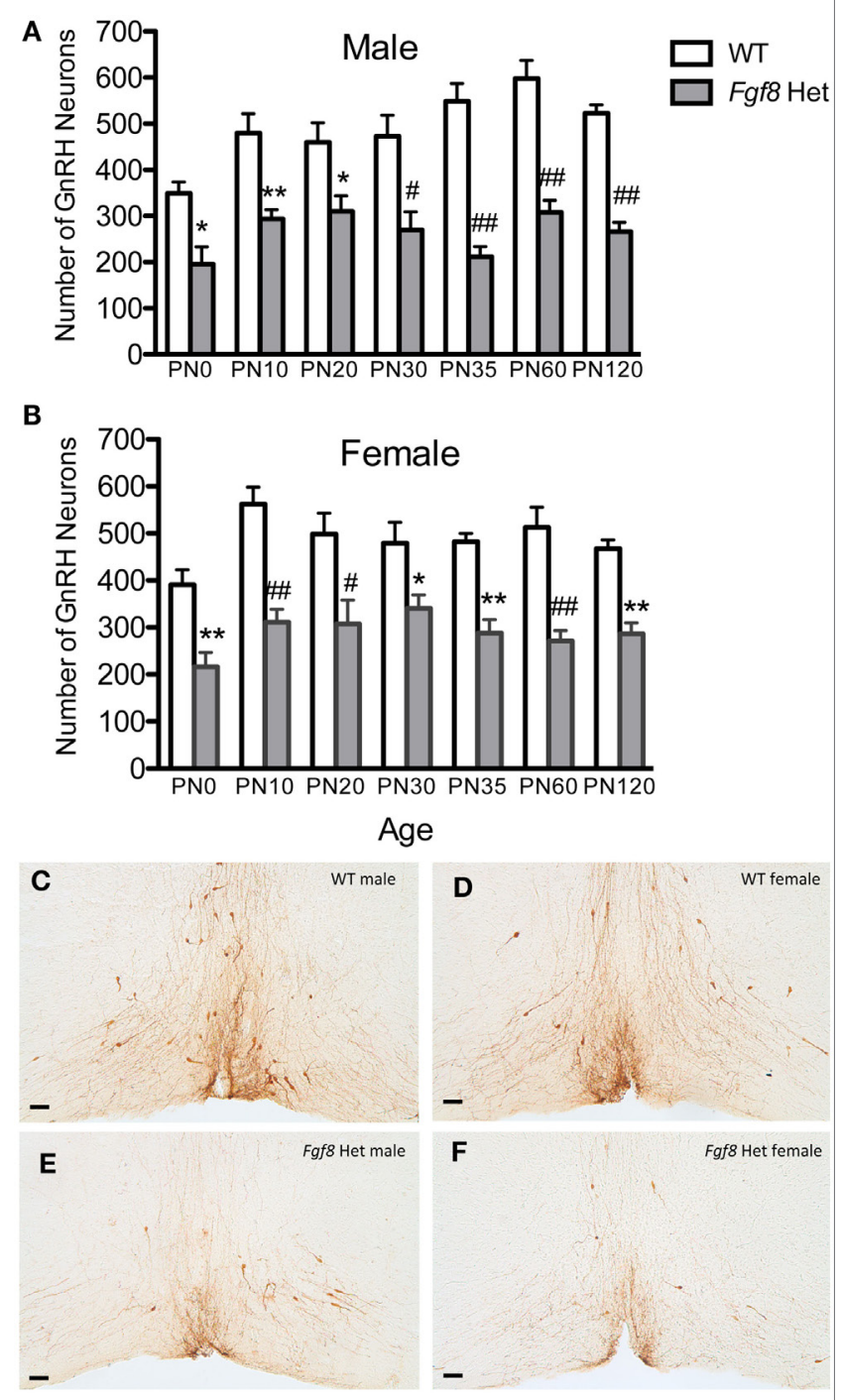

FIGURE 1 | Number of GnRH neurons in PN0-120 male (A) and female (B) WT and Fgf8 Het mice. In both sexes, GnRH neuron numbers in Fgf8 Het mice were consistently reduced compared to WT at all ages examined. Each bar $=$ mean \pm SEM; $N=5-6 .{ }^{\star} P<0.05 ;{ }^{*} P<0.01 ;{ }^{*} P<0.001$; ${ }^{\# \# P}<0.0001$ compared to WT of the same age. (C-F) Representative photomicrographs of $\mathrm{GnRH} I H C$ at the plane of OVLT in PN35 WT (C,D) and Fgf8 Het (E,F) male and female mice. Scale bar $=50 \mu \mathrm{m}$.

revealed a significant reduction of hypothalamic $\mathrm{GnRH}$ in $\mathrm{Fgf8}$ Het females only on PN60 (Figure 3B).

Female Fgf8 Het mice were previously shown to exhibit delayed puberty and slightly disrupted estrous cycle (26); however, male Fgf8 Het mice have not been examined for their reproductive parameters before, during, and after puberty. To address this, we initially compared testes/body mass and SV/ body mass ratios of WT and Fgf8 Het males at an age range that encompassed pre-pubertal (PN20), pubertal (PN35-40), and post-pubertal (PN60) periods. Two-way ANOVA revealed a significant effect of age $[F(3,46)=35.1 ; P<0.0001]$, but not

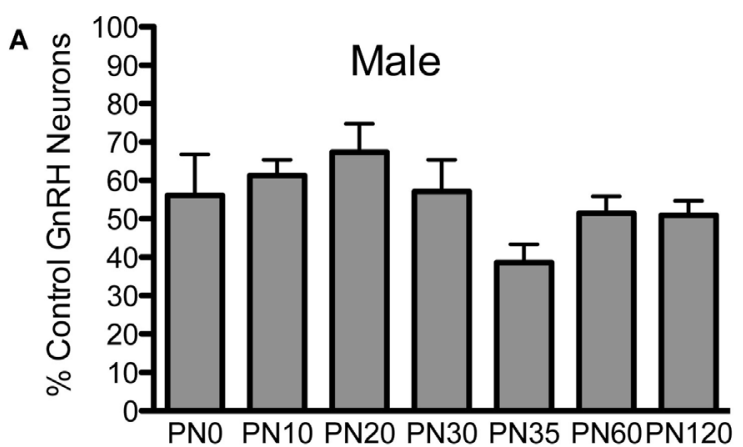

B

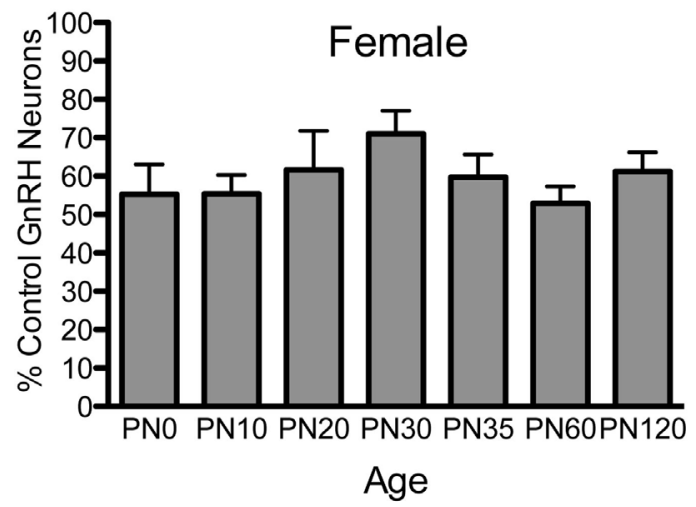

FIGURE 2 | Percent GnRH neurons in Fgf8 Het male (A) and female (B) mice. The number of $\mathrm{GnRH}$ neurons in each Fgf8 Het mouse was normalized against the mean of $\mathrm{GnRH}$ neurons in the same age and sex group. Each bar = mean \pm SEM; $N=5-6$. No differences among age groups were observed in either male or female Fgf8 Het mice.

genotype $[F(1,46)=3.69 ; P<0.061]$ or genotype $\times$ age interaction $[F(3,46)=0.09 ; P=0.96]$ on testes/body mass ratio (Figure 4A). Similarly, two-way ANOVA revealed a significant effect of age $[F(3,47)=146.1 ; P<0.0001]$, but not genotype $[F(1,47)=0.06 ; P<0.82]$ or genotype $\times$ age interaction $[F(3$, $47)=3.52 ; P=0.3]$ on SV/body mass ratio (Figure 4B). Overall, Fgf8 deficiency had little effects on testicular and SV growth over the ages examined.

Testicular growth alone was not indicative of spermatogenic capacity, thus we performed morphometric analyses on several testicular histological parameters in WT and Fgf8 Het males of different ages (Figure 5). Overall, two-way ANOVA revealed a significant effect of age on $\%$ ST with open lumen $[F(3$, $32)=11.39 ; P<0.0001$ ] (Figure 5A), \%ST with mature sperm $[F(3,32)=702.3 ; P<0.0001]$ (Figure 5B), ST diameter $[F(3$, $32)=143.1 ; P<0.0001]$ (Figure 5C), and ST luminal diameter $[F(3,32)=66.6 ; P<0.0001]$ (Figure 5D); however, no effects of genotype or genotype $\times$ age interaction were detected in any of these parameters, suggesting normal testicular function in Fgf8 Het males over the ages examined (Figures 5A-D).

Lastly, we measured plasma LH in PN35 and PN60 WT and Fgf8 males. These are the two ages in which we observed no (PN35) and a significant (PN60) effect of genotype on hypothalamic GnRH content (Figure 3A). No significant effects of 


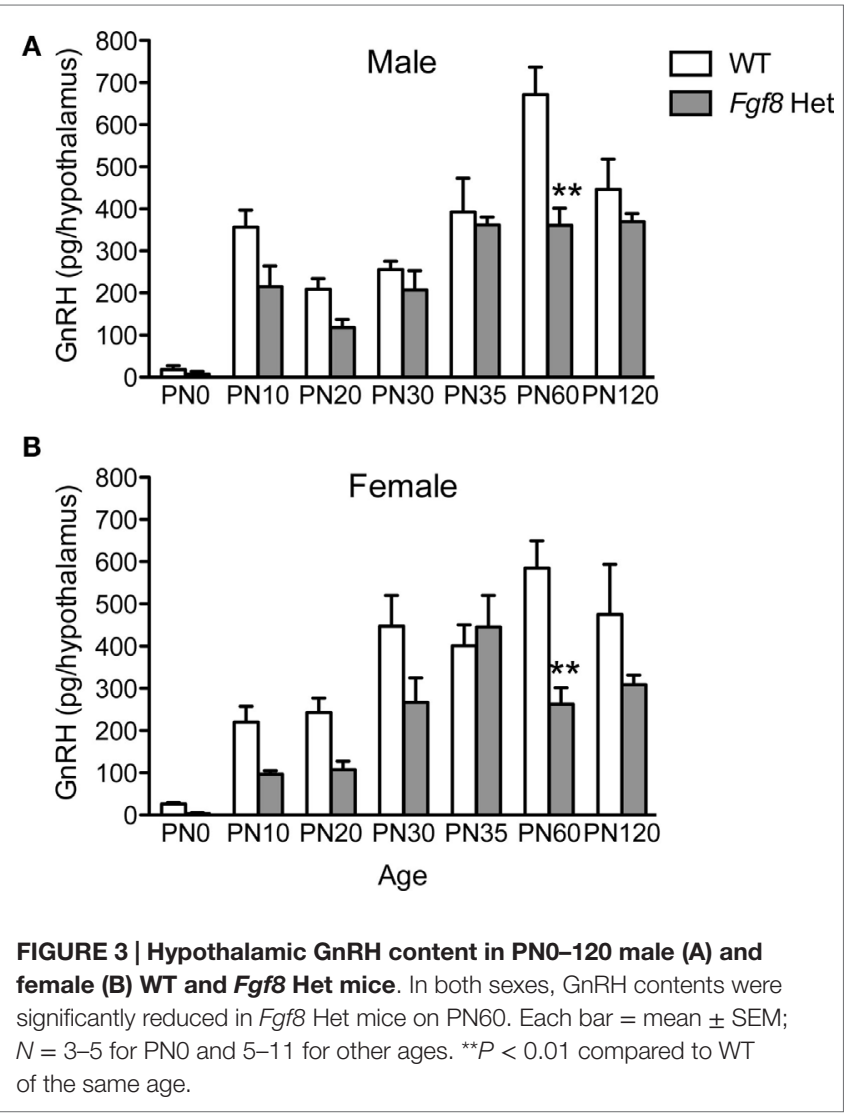

genotype, age, or age $\times$ genotype interaction on plasma $\mathrm{LH}$ were observed (Figure 6).

\section{Discussion}

Three key findings emerge from the present study. First, Fgf8 Het mice lost their GnRH neurons prenatally with no additional postnatal loss detected. Second, despite having an innately defective GnRH system, Fgf8 Het mice had normal levels of the $\mathrm{GnRH}$ peptide through most pre-pubertal and pubertal periods examined and exhibited GnRH peptide deficiency only at a specific age (PN60) after puberty. Lastly, pubertal transition, gaged by the mass of testes and SV and testicular morphology, was largely normal in Fgf8 Het males. Overall, our data underscored the inherent robustness of a neuroendocrine system that could compensate for the loss of almost half of its neuronal population with little reproductive consequence.

At present, the mechanism of prenatal GnRH neuronal loss in Fgf8 Het mice is unclear. Fgf8 Het mice had only $50 \% \mathrm{GnRH}$ neurons at birth $(20,22)$ but were reported to have a normal complement of GnRH neurons on embryonic day (E) 15.5 (22). The most parsimonious explanation for this discrepancy was that Fgf8 Het mice lost 50\% of their GnRH neurons between E15.5 and $\mathrm{PN} 0$ due to the failure of these neurons to migrate, survive, or remain differentiated. We considered migratory defect unlikely since most GnRH neurons in Fgf8 Het embryos were reported to reach the forebrain by E15.5 (22), an age in which the majority

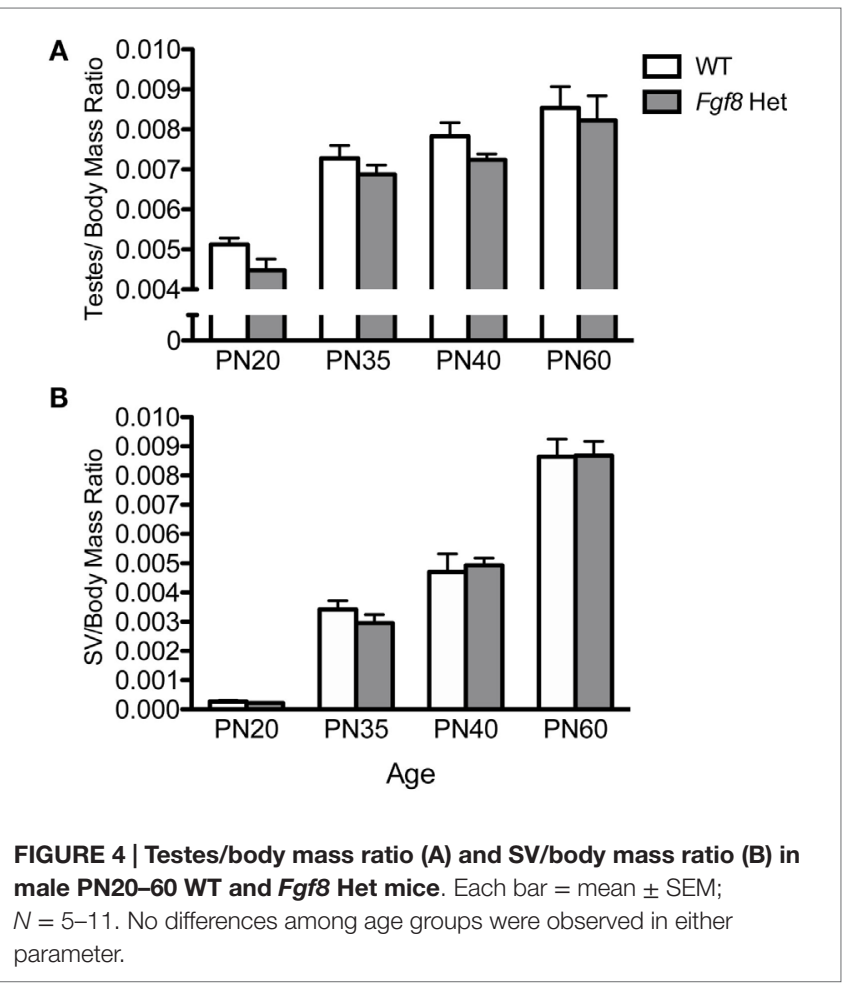

of neurons have completed their migration $(29,30)$. Of further interest, the loss of GnRH neurons observed in newborn Fgf8 Het mice persisted in all later ages examined, suggesting this loss was irreversible for the first four postnatal months and possibly the rest of the animals' lives. Moreover, no sex differences existed in the impact of $F g f 8$ deficiency on the GnRH neuronal population. These observations collectively supported the notion that the loss of GnRH neurons in Fgf8 Het mice was an irreversible developmental event that likely occurred after these neurons transitioned from the nose into the forebrain environment. Whether Fgf8 is needed to support the survival or differentiation of the developing $\mathrm{GnRH}$ neurons requires further interrogation, preferably using lineage-tracing techniques to permanently mark these neurons.

Although male and female Fgf8 Het mice exhibited consistent GnRH neuronal loss at all postnatal time points examined, a significant reduction in hypothalamic GnRH peptide levels was observed only on PN60 of both sexes (Figure 3). This observation carried several interesting implications, the foremost being that a defective GnRH system could largely compensate for the developmental GnRH deficit. The murine $\mathrm{GnRH}$ system was reported to be highly redundant, and a $\mathrm{GnRH}$ neuronal population that had been reduced by $90 \%$ could continue to sustain normal male reproduction (31). Our results suggested that Fgf8 Het mice may increase the level of bioavailable GnRH peptide via several mechanisms (see paragraph below) despite reduced GnRH neuron numbers. That said, Fgf8 Het females experienced delayed VO and first estrus (26), suggesting the compensation may not always be complete. Interestingly, Herbison et al. (31) also reported that the consequence of reduced $\mathrm{GnRH}$ neurons may be more deleterious in females than in males. At present, it 
A

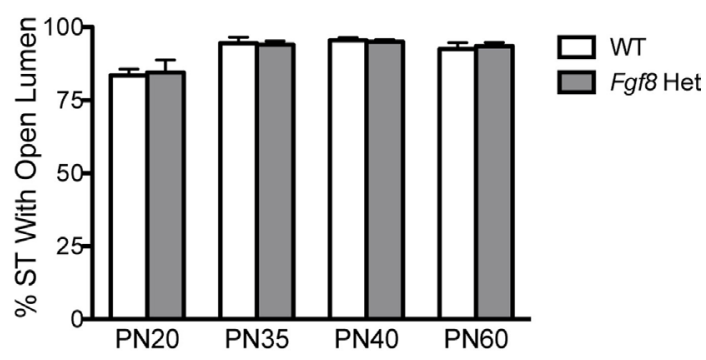

B

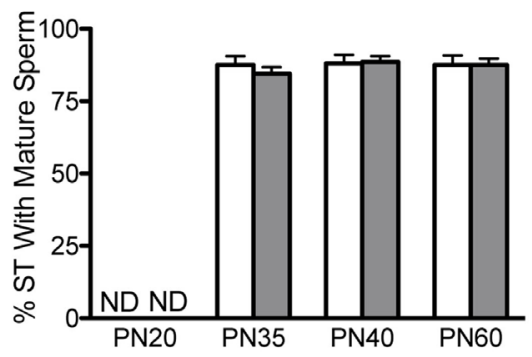

C

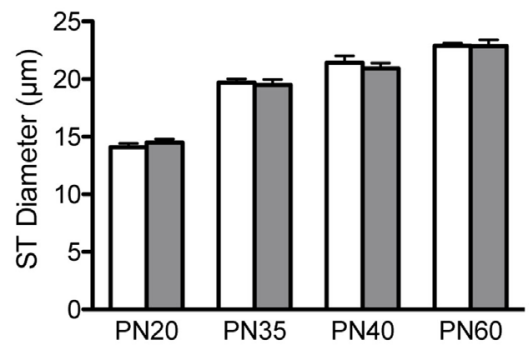

D

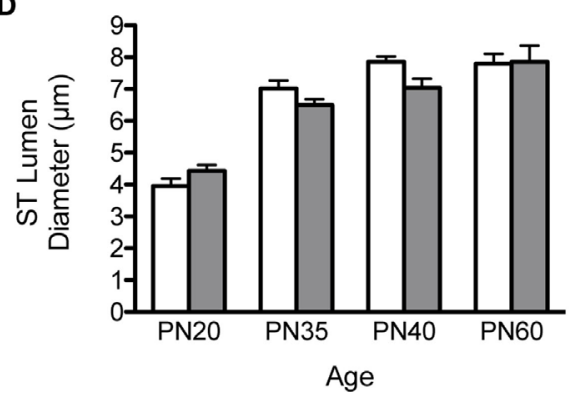

FIGURE 5 | Percent ST with open lumen (A), percent ST with mature sperm (B), ST diameter (C), and ST luminal diameter (D) in male PN20-60 WT and Fgf8 Het mice. Each bar = mean \pm SEM; $N=5$. $\mathrm{ND}=$ non-detectable. No differences among age groups were observed in any of the testicular parameters.

is unclear why only PN60 Fgf8 Het mice displayed a reduction in immunoassayable hypothalamic GnRH. Postnatally, hypothalamic GnRH peptide levels in mice peaked between PN30 and 40 (32) and GnRH transcript levels peaked between PN40 and 60 (33). This time course suggested that the GnRH system may have a robust synthetic machinery before and around puberty, but the synthetic drive plateaued after PN40. We hypothesize that Fgf8 Het mice may experience an earlier plateau than WT mice, leading to the observed pattern.

The mechanism leading to normal GnRH peptide levels in Fgf8 Het mice of most ages is unclear at present. The fewer GnRH

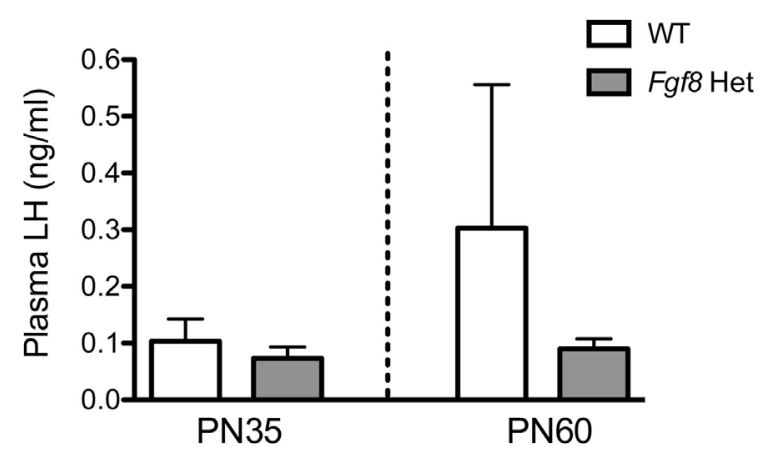

FIGURE 6 | Plasma LH in male PN35 and PN60 Fgf8 Het mice. Each bar $=$ mean $\pm \mathrm{SEM} ; N=6$ for PN35 and $N=10-11$ for PN60. No effects of genotype or age were observed.

neurons in Fgf8 Het mice could compensate by increasing mRNA levels via enhanced transcription and mRNA stability or by promoting translation efficiency and post-translational modification $(34,35)$. These mechanisms contribute to the accumulation of GnRH peptide and should be explored in the future. It is also interesting to note that the anteroventral periventricular (AVPV) kisspeptin system of Fgf8 Het females was downregulated on PN30 but normal at other pre- and post-pubertal periods (26). These results suggested that Fgf8 deficiency, besides reducing $\mathrm{GnRH}$ neurons, also reduced AVPV kisspeptin neurons agespecifically to delay female puberty. However, neither the AVPV nor the arcuate kisspeptin system in Fgf8 Het males had been examined, and their roles in safeguarding normal pubertal transition of these mice remain unclear.

Our analysis of testicular and SV/body mass ratios revealed no differences in the growth of these two reproductive organs between WT and Fgf8 Het mice. Since SV growth was androgendependent (36), these results suggested testicular steroidogenesis was not significantly altered in the mutant mice. Further analyses of testicular parameters such as \%ST with open lumen and mature sperm as well as ST total and luminal diameters also revealed no genotype differences before, during, and after pubertal transition (Figure 5). Consistent with these observations, the fertility of these mice was thought to be normal (25).

Previous studies reported the elimination of the entire $\mathrm{GnRH}$ neuronal population in $F g f 8$ homozygous hypomorphic mice (20, 22) despite the remaining presence of $\sim 50 \%$ functional Fgf 8 transcript, suggesting the exquisite sensitivity of the murine GnRH system to Fgf8. Despite the importance of Fgf8 in this system, Fgf8 Het mice have been consistently fertile in our colony. The lack of obvious male reproductive phenotype in Fgf8 Het mice may initially appear to contradict the reported subfertility and $\mathrm{HH}$ in some patients with $F g f 8$ haploinsufficiency $(15,24)$, but several explanations may account for this discrepancy. First, the reproductive phenotype of humans with $\mathrm{Fgf8}$ mutations has been difficult to predict due to variable penetrance and, in some cases, may be an oligogenic condition requiring additional disease genes to become fully manifested $(15,24,37)$. In other words, some $\mathrm{HH}$ patients with $\mathrm{Fgf8}$ haploinsufficiency may harbor 
additional mutations yet to be detected. Second, earlier studies transplanting GnRH neurons into hypogonadal ( $h p g$ ) mice (38) showed the successful transplantation of as few as 1-3 GnRH neurons could restore several reproductive parameters (39-41), suggesting the murine GnRH system is functionally robust and that 200-300 GnRH neurons in Fgf8 Het mice should adequately support reproduction. Third, we have not yet fully assessed the fertility of male Fgf8 Het mice, and such assessment may reveal more subtle defects. Indeed, although male $h p g$ mice transplanted with a small number of GnRH neurons exhibited elevated gonadotropin levels and underwent testicular and SV growth $(42,43)$, they did not exhibit normal gonadotropin and androgen levels and failed to ejaculate and impregnate females $(42,43)$. It would be interesting to assess if Fgf8 Het males exhibited normal mating frequency and fecundity. Lastly, the functional capacity of murine GnRH neurons may be highly dependent on the environmental condition. $\mathrm{HH}$ or suboptimal gonadal function stemming from reduced GnRH neurons could be reversed in mice simply by interactions with the opposite sex $(44,45)$, implicating high levels of functional plasticity. Future studies should assess

\section{References}

1. Wierman ME, Kiseljak-Vassiliades K, Tobet S. Gonadotropin-releasing hormone ( $\mathrm{GnRH}$ ) neuron migration: initiation, maintenance and cessation as critical steps to ensure normal reproductive function. Front Neuroendocrinol (2011) 32:43-52. doi:10.1016/j.yfrne.2010.07.005

2. Messina A, Giacobini P. Semaphorin signaling in the development and function of the gonadotropin hormone-releasing hormone system. Front Endocrinol (Lausanne) (2013) 4:133. doi:10.3389/fendo.2013.00133

3. Stevenson EL, Corella KM, Chung WC. Ontogenesis of gonadotropin-releasing hormone neurons: a model for hypothalamic neuroendocrine cell development. Front Endocrinol (Lausanne) (2013) 4:89. doi:10.3389/fendo.2013.00089

4. Mailleux AA, Tefft D, Ndiaye D, Itoh N, Thiery JP, Warburton D, et al. Evidence that SPROUTY2 functions as an inhibitor of mouse embryonic lung growth and morphogenesis. Mech Dev (2001) 102:81-94. doi:10.1016/ S0925-4773(01)00286-6

5. Furthauer M, Lin W, Ang SL, Thisse B, Thisse C. Sef is a feedback-induced antagonist of Ras/MAPK-mediated FGF signalling. Nat Cell Biol (2002) 4:170-4. doi:10.1038/ncb750

6. Leeksma OC, Van Achterberg TA, Tsumura Y, Toshima J, Eldering E, Kroes WG, et al. Human sprouty 4 , a new ras antagonist on $5 q 31$, interacts with the dual specificity kinase TESK1. Eur J Biochem (2002) 269:2546-56. doi:10.1046/j.1432-1033.2002.02921.x

7. Niehrs C, Meinhardt H. Modular feedback. Nature (2002) 417:35-6. doi:10.1038/417035a

8. Tsang M, Friesel R, Kudoh T, Dawid IB. Identification of Sef, a novel modulator of FGF signalling. Nat Cell Biol (2002) 4:165-9. doi:10.1038/ncb749

9. Dono R. Fibroblast growth factors as regulators of central nervous system development and function. Am J Physiol Regul Integr Comp Physiol (2003) 284:R867-81. doi:10.1152/ajpregu.00533.2002

10. Bottcher RT, Pollet N, Delius H, Niehrs C. The transmembrane protein XFLRT3 forms a complex with FGF receptors and promotes FGF signalling. Nat Cell Biol (2004) 6:38-44. doi:10.1038/ncb1082

11. Li C, Scott DA, Hatch E, Tian X, Mansour SL. Dusp6 (Mkp3) is a negative feedback regulator of FGF-stimulated ERK signaling during mouse development. Development (2007) 134:167-76. doi:10.1242/dev.02701

12. Taniguchi K, Ayada T, Ichiyama K, Kohno R, Yonemitsu Y, Minami Y, et al. Sprouty2 and Sprouty4 are essential for embryonic morphogenesis and regulation of FGF signaling. Biochem Biophys Res Commun (2007) 352:896-902. doi:10.1016/j.bbrc.2006.11.107

13. Hebert JM. FGFs: neurodevelopment's jack-of-all-trades - how do they do it? Front Neurosci (2011) 5:133. doi:10.3389/fnins.2011.00133 the fertility of $F g f 8$ Het mice under suboptimal environmental conditions, such as caloric restriction or psychosocial stress, to interrogate the functional outcome of a reduced GnRH system when challenged.

In conclusion, the present study reinforces the idea that a developmentally compromised GnRH system remains highly plastic. Except on PN60, the GnRH system in Fgf8 hypomorphic mice of various ages may compensate for the reduced GnRH neuron numbers by increasing the levels of bioavailable GnRH. The significant reduction in GnRH neuron number had no discernible impact on male testicular parameters or circulating $\mathrm{LH}$ as they transitioned through puberty. Overall, the present study showed that in spite of a significantly reduced GnRH system, compensatory mechanisms exist to drive sexual maturation and support adult gonadal function.

\section{Acknowledgments}

\section{This work was supported by NIH R01 HD042634.}

14. Dode C, Levilliers J, Dupont JM, De Paepe A, Le Du N, Soussi-Yanicostas $\mathrm{N}$, et al. Loss-of-function mutations in FGFR1 cause autosomal dominant Kallmann syndrome. Nat Genet (2003) 33:463-5. doi:10.1038/ng1122

15. Falardeau J, Chung WC, Beenken A, Raivio T, Plummer L, Sidis Y, et al. Decreased FGF8 signaling causes deficiency of gonadotropin-releasing hormone in humans and mice. J Clin Invest (2008) 118:2822-31. doi:10.1172/ JCI34538

16. Miraoui H, Dwyer AA, Sykiotis GP, Plummer L, Chung W, Feng B, et al. Mutations in FGF17, IL17RD, DUSP6, SPRY4, and FLRT3 are identified in individuals with congenital hypogonadotropic hypogonadism. Am J Hum Genet (2013) 92:725-43. doi:10.1016/j.ajhg.2013.04.008

17. Bachler M, Neubuser A. Expression of members of the Fgf family and their receptors during midfacial development. Mech Dev (2001) 100:313-6. doi:10.1016/S0925-4773(00)00518-9

18. Gill JC, Moenter SM, Tsai PS. Developmental regulation of gonadotropin-releasing hormone neurons by fibroblast growth factor signaling. Endocrinology (2004) 145:3830-9. doi:10.1210/en.2004-0214

19. Tsai PS, Moenter SM, Postigo HR, El Majdoubi M, Pak TR, Gill JC, et al. Targeted expression of a dominant-negative fibroblast growth factor (FGF) receptor in gonadotropin-releasing hormone $(\mathrm{GnRH})$ neurons reduces FGF responsiveness and the size of GnRH neuronal population. Mol Endocrinol (2005) 19:225-36. doi:10.1210/me.2004-0330

20. Chung WC, Moyle SS, Tsai PS. Fibroblast growth factor 8 signaling through fibroblast growth factor receptor 1 is required for the emergence of gonadotropin-releasing hormone neurons. Endocrinology (2008) 149:4997-5003. doi:10.1210/en.2007-1634

21. Chung WC, Matthews TA, Tata BK, Tsai PS. Compound deficiencies in multiple fibroblast growth factor signalling components differentially impact the murine gonadotrophin-releasing hormone system. J Neuroendocrinol (2010) 22:944-50. doi:10.1111/j.1365-2826.2010.02024.x

22. Forni PE, Bharti K, Flannery EM, Shimogori T, Wray S. The indirect role of fibroblast growth factor- 8 in defining neurogenic niches of the olfactory/GnRH systems. J Neurosci (2013) 33:19620-34. doi:10.1523/ JNEUROSCI.3238-13.2013

23. Zhang X, Ibrahimi OA, Olsen SK, Umemori H, Mohammadi M, Ornitz DM. Receptor specificity of the fibroblast growth factor family. The complete mammalian FGF family. J Biol Chem (2006) 281:15694-700. doi:10.1074/jbc. M601252200

24. Trarbach EB, Abreu AP, Silveira LF, Garmes HM, Baptista MT, Teles MG, et al. Nonsense mutations in FGF8 gene causing different degrees of human gonadotropin-releasing deficiency. J Clin Endocrinol Metab (2010) 95:3491-6. doi:10.1210/jc.2010-0176 
25. Meyers EN, Lewandoski M, Martin GR. An Fgf8 mutant allelic series generated by Cre- and Flp-mediated recombination. Nat Genet (1998) 18:136-41. doi:10.1038/ng0298-136

26. Tata BK, Chung WC, Brooks LR, Kavanaugh SI, Tsai PS. Fibroblast growth factor signaling deficiencies impact female reproduction and kisspeptin neurons in mice. Biol Reprod (2012) 86:119. doi:10.1095/ biolreprod.111.095992

27. Pak TR, Lynch GR, Tsai PS. Testosterone and estrogen act via different pathways to inhibit puberty in the male Siberian hamster (Phodopus sungorus). Endocrinology (2001) 142:3309-16. doi:10.1210/endo.142.8.8321

28. PakTR, Lynch GR, Ziegler DM, Lunden JB, Tsai PS. Disruption of pubertal onset by exogenous testosterone and estrogen in two species of rodents. Am J Physiol Endocrinol Metab (2003) 284:E206-12. doi:10.1152/ajpendo.00352.2002

29. Wray S. Development of luteinizing hormone releasing hormone neurones. J Neuroendocrinol (2001) 13:3-11. doi:10.1111/j.1365-2826.2001.00609.x

30. Wray S. Development of gonadotropin-releasing hormone-1 neurons. Front Neuroendocrinol (2002) 23:292-316. doi:10.1016/S0091-3022(02)00001-8

31. Herbison AE, Porteous R, Pape JR, Mora JM, Hurst PR. Gonadotropinreleasing hormone neuron requirements for puberty, ovulation, and fertility. Endocrinology (2008) 149:597-604. doi:10.1210/en.2007-1139

32. Halpin DM, Jones A, Fink G, Charlton HM. Postnatal ovarian follicle development in hypogonadal (hpg) and normal mice and associated changes in the hypothalamic-pituitary ovarian axis. J Reprod Fertil (1986) 77:287-96. doi:10.1530/jrf.0.0770287

33. Gore AC, Roberts JL, Gibson MJ. Mechanisms for the regulation of gonadotropin-releasing hormone gene expression in the developing mouse. Endocrinology (1999) 140:2280-7. doi:10.1210/en.140.5.2280

34. Gore AC, Roberts JL. Regulation of gonadotropin-releasing hormone gene expression in vivo and in vitro. Front Neuroendocrinol (1997) 18:209-45. doi:10.1006/frne.1996.0149

35. Wetsel WC, Srinivasan S. Pro-GnRH processing. Prog Brain Res (2002) 141:221-41. doi:10.1016/S0079-6123(02)41096-5

36. Fujii T. Roles of age and androgen in the regulation of sex accessory organs. Adv Sex Horm Res (1977) 3:103-37.

37. Sykiotis GP, Plummer L, Hughes VA, Au M, Durrani S, Nayak-Young S, et al. Oligogenic basis of isolated gonadotropin-releasing hormone deficiency. Proc Natl Acad Sci U S A (2010) 107:15140-4. doi:10.1073/pnas.1009622107

38. Mason AJ, Hayflick JS, Zoeller RT, Young WS III, Phillips HS, Nikolics K, et al. A deletion truncating the gonadotropin-releasing hormone gene is responsible for hypogonadism in the hpg mouse. Science (1986) 234:1366-71. doi:10.1126/science.3024317

39. Krieger DT, Perlow MJ, Gibson MJ, Davies TF, Zimmerman EA, Ferin M, et al. Brain grafts reverse hypogonadism of gonadotropin releasing hormone deficiency. Nature (1982) 298:468-71. doi:10.1038/298468a0

40. Gibson MJ, Charlton HM, Perlow MJ, Zimmerman EA, Davies TF, Krieger DT. Preoptic area brain grafts in hypogonadal (hpg) female mice abolish effects of congenital hypothalamic gonadotropin-releasing hormone (GnRH) deficiency. Endocrinology (1984) 114:1938-40. doi:10.1210/endo-114-5-1938

41. Gibson MJ, Krieger DT, Charlton HM, Zimmerman EA, Silverman AJ, Perlow MJ. Mating and pregnancy can occur in genetically hypogonadal mice with preoptic area brain grafts. Science (1984) 225:949-51. doi:10.1126/ science.6382608

42. Silverman AJ, Zimmerman EA, Gibson MJ, Perlow MJ, Charlton HM, Kokoris GJ, et al. Implantation of normal fetal preoptic area into hypogonadal mutant mice: temporal relationships of the growth of gonadotropin-releasing hormone neurons and the development of the pituitary/testicular axis. Neuroscience (1985) 16:69-84. doi:10.1016/0306-4522(85)90048-X

43. Livne I, Silverman AJ, Gibson MJ. Reversal of reproductive deficiency in the hpg male mouse by neonatal androgenization. Biol Reprod (1992) 47:561-7. doi:10.1095/biolreprod47.4.561

44. Good DJ, Porter FD, Mahon KA, Parlow AF, Westphal H, Kirsch IR. Hypogonadism and obesity in mice with a targeted deletion of the Nhlh2 gene. Nat Genet (1997) 15:397-401. doi:10.1038/ng0497-397

45. Rochester JR, Chung WC, Hayes T, Tsai PS. Opposite-sex housing reactivates the declining GnRH system in aged transgenic male mice with FGF signaling deficiency. Am J Physiol Endocrinol Metab (2012) 303:E1428-39. doi:10.1152/ ajpendo.00289.2012

Conflict of Interest Statement: The authors declare that the research was conducted in the absence of any commercial or financial relationships that could be construed as a potential conflict of interest.

Copyright (C) 2015 Zhang, Johnson and Tsai. This is an open-access article distributed under the terms of the Creative Commons Attribution License (CC BY). The use, distribution or reproduction in other forums is permitted, provided the original author(s) or licensor are credited and that the original publication in this journal is cited, in accordance with accepted academic practice. No use, distribution or reproduction is permitted which does not comply with these terms. 